\title{
A VOLUME STABILITY THEOREM ON TORIC MANIFOLDS WITH POSITIVE RICCI CURVATURE
}

\author{
FENG WANG \\ (Communicated by Lei $\mathrm{Ni}$ )
}

\begin{abstract}
In this short note, we will prove a volume stability theorem which says that if an $n$-dimensional toric manifold $M$ admits a $\mathbb{T}^{n}$ invariant Kähler metric $\omega$ with Ricci curvature no less than 1 and its volume is close to the volume of $\mathbb{C P}^{n}, M$ is bi-holomorphic to $\mathbb{C P}^{n}$.
\end{abstract}

\section{INTRODUCTION}

Understanding the geometry of manifolds under various curvature conditions is fundamental. In Riemannian geometry, we have Bishop-Gromov's volume comparison if the Ricci curvature of the manifold is bounded from below. Using this theorem and some techniques in comparison geometry, Colding proved the following result ( $[\underline{5})$ :

Theorem 1.1. Given $\epsilon>0$, there exists $\delta=\delta(n, \epsilon)>0$ such that, if an $n$ dimensional manifold $M$ has $\operatorname{Ric}_{M} \geq n-1$ and $\operatorname{Vol}(M)>\operatorname{Vol}\left(\mathbb{S}^{n}\right)-\delta$, then $d_{G H}\left(M, \mathbb{S}^{n}\right)<\epsilon$.

Here $d_{G H}$ denotes the Gromov-Hausdorff distance between Riemannian manifolds. By another theorem of Colding (see the appendix in [4]), we know that $M$ is in fact diffeomorphic to $\mathbb{S}^{n}$.

Natural questions are how to get a more useful version of Bishop-Gromov's volume comparison theorem in Kähler geometry and how to state a theorem analogous to the one above. Because we have more structures on the manifold, the volume comparison with space form is not sharp: see [8] for an improvement of the local volume comparison for Kähler manifolds with Ricci curvature bounded from below. More recently, Berman and Berndtsson considered toric manifolds with positive Ricci curvature in [2] and [3], and they proved:

Theorem 1.2. Suppose that $(M, \omega)$ is a smooth $n$-dimensional toric variety with $\mathbb{T}^{n}$ invariant Kähler form $\omega$ such that Ric $\omega \geq \omega$; then we have

$$
\operatorname{Vol}(M) \leq \operatorname{Vol}\left(\mathbb{C P}^{n}\right) .
$$

In fact, their theorem holds if the manifold admits a $\mathbb{C}^{*}$ action with finite fixed points and the metric is $\mathbb{S}^{1}$ invariant (see [3]). The theorem of Berman and Berndtsson partially answered a conjecture in $[10$.

Received by the editors November 3, 2012 and, in revised form, February 19, 2013.

2010 Mathematics Subject Classification. Primary 53C23; Secondary 53C55.

Key words and phrases. Differential geometry. 
Conjecture. Any n-dimensional toric Fano manifold $X$ that admits a KählerEinstein metric has anticanonical degree $\left(-K_{X}\right)^{n} \leq(n+1)^{n}$, with equality only for $\mathbb{C P}^{n}$.

In this short note, we will determine when the equality holds in Theorem 1.2 So the above conjecture is completely solved. More precisely, we can prove a rigidity and stability theorem as follows:

Theorem 1.3. The equality in Theorem 1.2 holds if and only if $(M, \omega)$ is isometric to $\left(\mathbb{C P}^{n}, \omega_{F S}\right)$. Moreover, there exists a positive number $\epsilon$ which depends only on $n$ such that if $M$ is a toric manifold with a $\mathbb{T}^{n}$ invariant metric $\omega$ satisfying Ric $\omega \geq \omega$ and

$$
\operatorname{Vol}(M) \geq(1-\epsilon) \operatorname{Vol}\left(\mathbb{C P}^{n}\right),
$$

$M$ is bi-holomorphic to $\mathbb{C P}^{n}$.

In [3], Berman and Berndtsson applied a Moser-Trudinger typed inequality established in [1] to prove Theorem 1.2. But so far we can't prove the rigidity using this analytic method. Inspired by the combinatoric proof by Bo'az Klartag for the Kähler-Einstein case in [3], we will apply the Grunbaum's inequality ([7]) to prove our theorem. In order to use this inequality we should know the position of the barycenter of the moment polytope of $(M, \omega)$. We will use the Ricci curvature condition to achieve this. More detailed analysis gives us the rigidity and stability.

\section{Preliminaries}

At first, we give some basic materials of toric manifolds which are used in our proof. Here a toric manifold means a Kähler manifold $(M, \omega)$ containing $\left(\mathbb{C}^{*}\right)^{n}$ as a dense subset such that the standard action of $\left(\mathbb{C}^{*}\right)^{n}$ on itself extends to a holomorphic action on $M$. In general we suppose that the metric is $\mathbb{T}^{n}$ invariant and we can consider the moment map of $\left(M, \omega, \mathbb{T}^{n}\right)$.

Definition 2.1. A polytope $P \subseteq \mathbb{R}^{n}$ is called a Delzant polytope if each vertex is contained in exactly $n$ facets, and the normals of the $n$ facets containing a given vertex form an integral basis of $\mathbb{Z}^{n}$.

The image of the moment map above should be a Delzant polytope according to a theorem of Delzant $(\underline{6}])$ :

Theorem 2.2. Each Delzant polytope gives rise to a symplectic manifold $(M, \omega)$ with an action of $\mathbb{T}^{n}$ that preserves $\omega$, and all such symplectic manifolds arise this way.

In fact, congruent polytopes correspond to isomorphic toric symplectic manifolds.

Using the embedding of $\left(\mathbb{C}^{*}\right)^{n}$ in $M$, we set:

$$
\iota:\left(\mathbb{C}^{*}\right)^{n} \rightarrow M, \iota^{*} \omega=\sqrt{-1} \partial \bar{\partial} u .
$$

In toric coordinates: $\exp \left(x_{i}\right)=\left|z_{i}\right|^{2}\left(z_{i}\right.$ are holomorphic coordinates in $\left.\left(\mathbb{C}^{*}\right)^{n}\right)$, the invariance of $\omega$ means $u$ is a function of $x_{i}$. Then the image of $\nabla u=\left(\frac{\partial u}{\partial x_{i}}\right)_{1 \leq i \leq n}$ will be a moment map of $(M, \omega)$. 
Given a toric manifold $(M, \omega)$, two moment maps may differ by a constant vector. When we choose a basis of group $\left(\mathbb{C}^{*}\right)^{n}$, these two moment polytopes differ by a translation. A change of basis of group $\left(\mathbb{C}^{*}\right)^{n}$ corresponds to a change of the integral basis of $\mathbb{Z}^{n}$, so it transforms Delzant polytopes to Delzant polytopes. The polytope also changes if we choose another $\mathbb{T}^{n}$ invariant Kähler metric on $M$ with the same complex structure; i.e., we choose another symplectic form compatible with the fixed complex structure. This can be described in the following way: we denote the moment polytope by

$$
P=\left\{x \mid\left\langle l_{i}, x\right\rangle \geq \lambda_{i}, 1 \leq i \leq N, x \in \mathbb{R}^{n}, l_{i} \in \mathbb{Z}^{n}, \lambda_{i} \in \mathbb{R}\right\} .
$$

Then only $\lambda_{i}(1 \leq i \leq n)$ change while $l_{i}(1 \leq i \leq n)$ remain the same since they are just related to the complex structure (see [9], 11]). Using the description above, changing the symplectic form corresponds to changing the potential function $u$ on $\mathbb{R}^{n}$.

When the manifold is a Fano variety with $\omega \in 2 \pi c_{1}(M)$, we can get a moment polytope $P$ such that $\lambda_{i}$ are all equal to -1 . This can be realized in the following way (see [9]): choose a potential $u$ of $\omega$ such that

$$
\left|\ln \operatorname{det} u_{i j}+u\right| \text { is bounded in } \mathbb{R}^{n} \text {; }
$$

then the image of $\nabla u$ will be such a polytope $P$ with $\lambda_{i}=-1(1 \leq i \leq n)$.

Because the normal vectors of the facets passing any point form an integral basis, we can do a coordinate transformation to change these vectors to the standard basis $e_{k}=(0,0, \ldots, 1,0, \ldots, 0)$ with 1 placed at position $k$. We can write this transformation as follows: choosing a vertex $p \in P$ with $l_{i}(1 \leq i \leq n)$ as normal vectors of the facets passing $p$, we can form an affine map:

$$
x \mapsto\left(\left\langle l_{i}, x\right\rangle\right)_{1 \leq i \leq n},
$$

which transforms $p$ to $(-1,-1, \ldots,-1)$ and the polytope to

$$
\tilde{P}=\left\{x \mid\left\langle\tilde{l}_{i}, x\right\rangle \geq-1,1 \leq i \leq N, x \in \mathbb{R}^{n}, \tilde{l}_{i} \in \mathbb{Z}^{n}, \tilde{l}_{k}=e_{k}, 1 \leq k \leq n\right\} .
$$

There are only a finite many such polytopes in a given dimension.

According to Mabuchi's theorem ([9]), we know that for a Kähler-Einstein manifold, the origin is the barycenter of $P$. We will prove a similar property of the barycenter of the moment map of a toric manifold admitting $\omega$ with Ric $\omega \geq \omega$.

\section{Proof of Theorem 1.3}

At first we give a lemma which deals with the volume of some specific kind of polytopes. Let $Q$ be the simplex spanned by

$$
(n+1,0,0, \ldots, 0),(0, n+1,0,0, \ldots, 0), \ldots,(0,0, \ldots, 0, n+1) .
$$

Recall that Grunbaum's inequality ([7]) says that if $P$ is a convex body, and $K$ denotes the intersection of $P$ with an affine half-space defined by one side of a hyperplane $H$ passing through the barycenter of $P$, then

$$
\operatorname{Vol}(P) \leq\left(\frac{n+1}{n}\right)^{n} \operatorname{Vol}(K) .
$$

Let $F$ denote the simplex spanned by

$$
(n, 0,0, \ldots, 0),(0, n, 0,0, \ldots, 0), \ldots,(0,0, \ldots, 0, n) .
$$


We have the following lemma.

Lemma 3.1. If the barycenter of a polytope $P$ in the first quadrant lies inside $F$,

$$
\operatorname{Vol}(P) \leq \operatorname{Vol}(Q) \text {. }
$$

Moreover, if the equality holds, $P$ is coincident with $Q$.

Proof. The first statement can be seen from Grunbaum's theorem above: the corresponding $K \subseteq F$. For the second statement, let $X=P \backslash Q, Y=Q \backslash P$ and choose a coordinate system $s_{i}$ with the barycenter as the origin and $(1,1, \ldots, 1)$ as the first axis. Then we have

$$
\int_{P} s_{1} d V \leq \int_{Q} s_{1} d V=0, \quad \int_{X} s_{1} d V \leq \int_{Y} s_{1} d v .
$$

But since

$$
s_{1}(x) \geq s_{1}(y) \text { for } x \in X \text { and } y \in Y,
$$

both $X$ and $Y$ should be empty.

In order to apply this lemma to the moment polytope $P$ of $(M, \omega)$, we should know how to place $P$ and where the barycenter is. We are going to use the toric structure on $M$ and explore the Ricci curvature condition.

Under the conditions of Theorem 1.3, we can write Ric $\omega=\omega+\beta$ where $\beta$ is a semi-positive 1-1 form. In $\left(\mathbb{C}^{*}\right)^{n}$, we can choose $u$ such that $\omega=\sqrt{-1} \partial \bar{\partial} u$. In toric coordinates: $\left|z_{i}\right|^{2}=\exp \left(x_{i}\right)$, we set:

$$
v=-\ln \operatorname{det} u_{i j}-u \text {. }
$$

Using the formula of Ricci curvature and $\frac{\partial^{2} u}{\partial z_{i} \partial \bar{z}_{j}}=\frac{\partial^{2} u}{\partial x_{i} \partial x_{j}} \frac{1}{z_{i}} \frac{1}{\overline{z_{j}}}$, we see that

$$
\sqrt{-1} \partial \bar{\partial} v=\operatorname{Ric} \omega-\omega=\beta .
$$

As $\beta$ is semi-positive, $v$ is a convex function.

From the following equalities:

$$
\ln \operatorname{det}(u+v)_{i j}+u+v=\ln \operatorname{det}(u+v)_{i j}-\ln \operatorname{det} u_{i j}=\ln \frac{(\operatorname{Ric} \omega)^{n}}{\omega^{n}},
$$

we know that $\ln \operatorname{det}(u+v)_{i j}+u+v$ is bounded, so $\nabla(u+v)$ will be a moment map of $(M, \operatorname{Ric} \omega)$. Denote the image of $\nabla(u+v)$ by $L$. As illustrated in section 2 , we can suppose that $(-1,-1, \ldots,-1)$ is a vertex of $L$ and the facets passing it are parallel to coordinate hyperplanes, respectively:

$$
L=\left\{y \mid\left\langle l_{i}, y\right\rangle \geq-1,1 \leq i \leq N, y \in \mathbb{R}^{n}, l_{i} \in \mathbb{Z}^{n}, l_{k}=e_{k}, 1 \leq k \leq n\right\} .
$$

The gradient of $u$ will be a moment of $(M, \omega)$. We denote the image of $\nabla u$ by $P$. Without changing $u+v$, we can add a linear function to $u$ and subtract the same one from $v$. This corresponds to a translation of $P$. As we have said above, $P$ can be obtained from $L$ by parallel movement of the facets. So we can translate $P$ so that $(-1,-1, \ldots,-1)$ is a vertex of $P$ and the facets passing this vertex are parallel to coordinate hyperplanes like $L$ :

$$
P=\left\{y \mid\left\langle l_{i}, y\right\rangle \geq \lambda_{i}, 1 \leq i \leq N, y \in \mathbb{R}^{n}, l_{i} \in \mathbb{Z}^{n}, l_{k}=e_{k}, \lambda_{k}=-1,1 \leq k \leq n\right\} .
$$

Such a pair of polytopes $(P, L)$ is called an adapted pair of $(M, \omega)$. 
Lemma 3.2. For an adapted pair $(P, L)$, the coordinates of the barycenter of $P$ are all nonpositive.

Proof.

$$
\lim _{x_{i} \rightarrow-\infty} \frac{\partial v}{\partial x_{i}}=\lim _{x_{i} \rightarrow-\infty} \frac{\partial(u+v)}{\partial x_{i}}-\lim _{x_{i} \rightarrow-\infty} \frac{\partial u}{\partial x_{i}}=(-1)-(-1)=0
$$

for any $i$ and fixed $x_{j}(1 \leq j \leq n, j \neq i)$. Because $v$ is a convex function we know that all the partial derivatives of $v$ are nonnegative. Denoting the coordinates of the barycenter by $a_{i}$, we have

$$
\begin{gathered}
\operatorname{det} u_{i j}=\exp (-u-v), \frac{\partial v}{\partial x_{i}} \geq 0 \\
a_{i}=\int_{P} y_{i} d V=\int_{R^{n}} \frac{\partial u}{\partial x_{i}} \operatorname{det} u_{i j} d x \leq \int_{R^{n}} \frac{\partial(u+v)}{\partial x_{i}} \exp (-u-v) d x=0 .
\end{gathered}
$$

The last inequality is the statement of the lemma.

Proof of Theorem 1.3. Using the notations above, we do a translation which moves $(-1,-1, \ldots,-1)$ to the origin. Then $P$ will be a polytope inside the first quadrant with barycenter inside $F$ by the second lemma. The rigidity follows from this together with the assumption that $\operatorname{Vol}(P)=\operatorname{Vol}(Q)$ by the first lemma.

Now we consider the stability. Suppose the statement doesn't hold; then there is a sequence of manifolds $\left(M_{i}, \omega_{i}\right)(i=1,2,3, \ldots)$ with volume converging to $\operatorname{Vol}\left(\mathbb{C P}^{n}\right)$ and with none holomorphic to $\mathbb{C P}^{n}$.

Construct adapted pairs $\left(P_{i}, L_{i}\right)$ of $\left(M_{i}, \omega_{i}\right)(i=1,2,3, \ldots)$. Because there are only finitely many such $L$, one of them appears infinitely times. We denote it by $B$ and select these $P_{i}$ corresponding to $B$. These $P_{i}$ as moment polytopes of different symplectic classes can be obtained from $B$ by parallel movement of $B$ 's facets towards the interior. So $P_{i}$ can be determined by $N$ real numbers $\lambda_{i}$ such that $n$ of them are always -1 . This gives us a correspondence:

$$
P_{i} \leftrightarrow \lambda^{(i)} \in \mathbb{R}^{N-n} .
$$

Because $P_{i}$ are inside $B$, these vectors in $\mathbb{R}^{N-n}$ are bounded. We can choose a convergent subsequence, and the limit corresponds to a polytope $P_{\infty} \cdot \operatorname{Vol}\left(P_{\infty}\right)=$ $\operatorname{Vol}(Q)$ and the coordinates of the barycenter of $P_{\infty}$ are all nonpositive. According to the first lemma, $P_{\infty}$ should be isomorphic to $Q$ by a translation. We are going to show that $B=P_{\infty}$ : Since $P_{i} \subseteq B$, we have $P_{\infty} \subseteq B$. If $P_{\infty} \neq B$, the integral points in the interior of the facet of $P_{\infty}$ opposite $(-1,-1, \ldots,-1)$ will be contained in the interior of $B$. But there is only one integral point in the interior of $B$, so we must have $B=P_{\infty}$.

We assumed that $M_{i}$ are not holomorphic to $\mathbb{C P}^{n}$, but now $B$ just differs from $Q$ by a translation. Because congruent polytopes give rise to isomorphic toric varieties, these $M_{i}$ are all holomorphic to $\mathbb{C P}^{n}$. This is a contradiction, so our theorem is proved.

\section{ACKNOWLEDGEMENT}

The author is grateful to his advisor, Professor Zhu Xiaohua, for many helpful conversations. 


\section{REFERENCES}

[1] R.J. Berman and B. Berndtsson, Moser-Trudinger type inequalities for complex MongeAmpère operators and Aubins hypothèse fondamentale, arXiv:1109.1263.

[2] R.J. Berman and B. Berndtsson, The projective space has maximal volume among all toric Käler-Einstein manifolds, arXiv:1112.4445.

[3] R.J. Berman and B. Berndtsson, The volume of Kähler-Einstein Fano varieties and convex bodies, arXiv:1204.1308.

[4] Jeff Cheeger and Tobias H. Colding, On the structure of spaces with Ricci curvature bounded below. I, J. Differential Geom. 46 (1997), no. 3, 406-480. MR1484888 (98k:53044)

[5] Tobias H. Colding, Shape of manifolds with positive Ricci curvature, Invent. Math. 124 (1996), no. 1-3, 175-191, DOI 10.1007/s002220050049. MR1369414 (96k:53067)

[6] Thomas Delzant, Hamiltoniens périodiques et images convexes de l'application moment (French, with English summary), Bull. Soc. Math. France 116 (1988), no. 3, 315-339. MR 984900 (90b:58069)

[7] B. Grünbaum, Partitions of mass-distributions and of convex bodies by hyperplanes., Pacific J. Math. 10 (1960), 1257-1261. MR.0124818 (23 \#A2128)

[8] G. Liu, Kahler manifolds with Ricci curvature lower bound, arXiv:1108.4232.

[9] Toshiki Mabuchi, Einstein-Kähler forms, Futaki invariants and convex geometry on toric Fano varieties, Osaka J. Math. 24 (1987), no. 4, 705-737. MR927057 (89e:53074)

[10] Benjamin Nill and Andreas Paffenholz, Examples of Kähler-Einstein toric Fano manifolds associated to non-symmetric reflexive polytopes, Beitr. Algebra Geom. 52 (2011), no. 2, 297304, DOI 10.1007/s13366-011-0041-y. MR2842630

[11] Tadao Oda, Convex bodies and algebraic geometry, Ergebnisse der Mathematik und ihrer Grenzgebiete (3) [Results in Mathematics and Related Areas (3)], vol. 15, Springer-Verlag, Berlin, 1988. An introduction to the theory of toric varieties; Translated from the Japanese. MR.922894(88m:14038)

School of Mathematical Science, Beijing University, Beijing, People's Republic of China 100871

E-mail address: fengwang232@gmail.com 